\title{
Management of varicella zoster virus retinitis in AIDS
}

Ramana S Moorthy, David V Weinberg, Steven A Teich, Brian B Berger, John T Minturn, Sanjiv Kumar, Narsing A Rao, Susan M Fowell, Isaac A Loose, Lee M Jampol

Associated

Vitreoretinal and

Uveitis Consultants, Indianapolis, USA

R S Moorthy

Northwestern

University, Chicago,

USA

D V Weinberg

L M Jampol

Mount Sinai Medical Center, New York, USA

S A Teich

Retina Consultants of Texas, Austin, USA

B B Berger

Midwest Eye Institute, Indianapolis, USA

$\mathrm{J}$ T Minturn

Doheny Eye Institute, Los Angeles, USA

S Kumar

N A Rao

Northern Illinois Retina Ltd, Rockford, USA

$S M$ Fowell

I A Loose

Correspondence to: David V Weinberg, MD, Department of Ophthalmology,

Northwestern University Medical School, Suite 440, 645 N Michigan Avenue, Chicago, IL 60611, USA

Accepted for publication 4 November 1996

\begin{abstract}
Aims/background-Varicella zoster virus retinitis (VZVR) in patients with AIDS, also called progressive outer retinal necrosis (PORN), is a necrotising viral retinitis which has resulted in blindness in most patients. The purposes of this study were to investigate the clinical course and visual outcome, and to determine if the choice of a systemic antiviral therapy affected the final visual outcome in patients with VZVR and AIDS.

Methods-A review of the clinical records of 20 patients with VZVR from six centres was performed. Analysis of the clinical characteristics at presentation was performed. Kruskall-Wallis non-parametric one way analysis of variance (KWAOV) of the final visual acuities of patients treated with acyclovir, ganciclovir, foscarnet, or a combination of foscarnet and ganciclovir was carried out.
\end{abstract}

Results-Median follow up was 6 months (range 1.3-26 months). On presentation, 14 of 20 patients $(70 \%)$ had bilateral disease, and $75 \%$ (15 of 20 patients) had previous or concurrent extraocular manifestations of VZV infection. Median initial and final visual acuities were $20 / 40$ and hand movements, respectively. Of 39 eyes involved, 19 eyes (49\%) were no light perception at last follow up; 27 eyes $(69 \%)$ developed rhegmatogenous retinal detachments. Patients treated with combination ganciclovir and foscarnet therapy or ganciclovir alone had significantly better final visual acuity than those treated with either acyclovir or foscarnet (KWAOV: $p=0.0051$ ).

Conclusions-This study represents the second largest series, the longest follow up, and the first analysis of visual outcomes based on medical therapy for AIDS patients with VZVR. Aggressive medical treatment with appropriate systemic antivirals may improve long term visual outcome in patients with VZVR. Acyclovir appears to be relatively ineffective in treating this disease.

(Br f Ophthalmol 1997;81:189-194)

Varicella zoster virus retinitis (VZVR) in AIDS, also known as progressive outer retinal necrosis (PORN), is a clinically distinct necrotising retinitis syndrome caused by varicella zoster virus which occurs in immunocompromised patients. It has a rapid and progressive course leading to blindness in most cases. ${ }^{1-4}$ At the time of presentation, eyes demonstrate minimal clinical signs of inflammation. Most patients have a progressive relentless downhill course resulting in blindness. In one recent large series of patients with PORN, $67 \%$ of eyes had final visual acuity of no light perception (NLP) after a median follow up period of 4 weeks, and retinal detachment occurred in $70 \%$ of involved eyes. ${ }^{3}$ Although these studies have looked at the course and visual outcome of patients with VZVR, they did not address differences in visual outcome of patients treated with different antiviral regimens. We have performed a review of 20 patients from six centres with the diagnosis of AIDS and VZVR. We evaluated the long term visual outcome of these patients reviewing the medical therapies that were used. To our knowledge this is the second largest reported series of patients with PORN. We report the longest follow up of patients, and have analysed for visual outcomes with different treatment strategies.

\section{Materials and methods}

We performed a review of 20 consecutive patients with the diagnosis of VZVR and AIDS from six centres between April 1991 and April 1994. These included Northwestern University Medical School in Chicago, Mount Sinai School of Medicine, New York, Methodist Hospital in Indianapolis, Retina Consultants of Texas, Austin, University of Southern California/Doheny Eye Institute/Los Angeles County Hospital, Los Angeles, and Northern Illinois Retina Ltd, Rockford, Illinois. All patients met the diagnostic criteria for PORN described by Engstrom and associates. ${ }^{3}$ Thirty nine eyes of 20 patients were included in the study. The patients and involved eyes were evaluated based on the demographic variables: age, race, sex, and presenting CD4 lymphocyte count, and the characteristics of clinical presentation: unilateral or bilateral involvement, macular or peripheral presentation, evidence of extraocular manifestations of VZV infection (see Tables 1 and 2).

Antiviral therapy at the time of presentation and subsequent addition or change in antiviral regimen were recorded for each patient. Although treatment strategies varied among investigators we classified treatment based on the maximum medical therapy used for each patient. Maximum therapy was defined, from least to most aggressive, as follows: intravenous acyclovir (at least $10 \mathrm{mg} / \mathrm{kg}$ intravenously every 
Table 1 Summary of clinical characteristics of 20 patients with varicella zoster virus (VZV) retinitis

\begin{tabular}{|c|c|c|c|c|c|c|c|c|}
\hline Patient & $\begin{array}{l}\text { Age } \\
\text { (years) }\end{array}$ & Race & $\operatorname{Sex}$ & $\begin{array}{l}\text { CD4 } \\
\left(\text { cells } \times 10^{6} / l\right)\end{array}$ & $\begin{array}{l}\text { Extraocular } \\
\text { manifestations }\end{array}$ & $\begin{array}{l}\text { Unilateral/ } \\
\text { bilateral }\end{array}$ & $\begin{array}{l}\text { Time to } 2 \text { nd eye } \\
\text { involvement } \\
\text { (days) }\end{array}$ & $\begin{array}{l}\text { Duration of } \\
\text { follow up } \\
\text { (days) }\end{array}$ \\
\hline 1 & 31 & Black & M & 4 & VZV & Unilateral & 29 & 50 \\
\hline 2 & 43 & White & M & 168 & VZV & Unilateral & - & 30 \\
\hline 3 & 31 & Black & M & 8 & VZV & Bilateral & 0 & 180 \\
\hline 4 & 28 & Hispanic & M & 21 & VZV & Unilateral & 17 & 505 \\
\hline 5 & 35 & Black & $\mathrm{F}$ & 9 & VZV & Unilateral & 17 & 150 \\
\hline 6 & 30 & White & M & 6 & VZV & Bilateral & 0 & 214 \\
\hline 7 & 42 & White & M & 8 & None & Bilateral & 0 & 240 \\
\hline 8 & 51 & Black & $M$ & 3 & VZV & Bilateral & 0 & 75 \\
\hline 9 & 38 & White & M & 39 & VZV & Unilateral & 1 & 790 \\
\hline 10 & 37 & Black & $M$ & 120 & VZV & Bilateral & 0 & 270 \\
\hline 11 & 36 & White & M & & VZV & Bilateral & 0 & 140 \\
\hline 12 & 45 & White & $M$ & 41 & None & Bilateral & 0 & 72 \\
\hline 13 & 36 & Hispanic & $M$ & 69 & HSV & Bilateral & 0 & 330 \\
\hline 14 & 36 & Hispanic & $\mathrm{F}$ & 31 & HSV & Bilateral & 0 & 162 \\
\hline 15 & 44 & Black & $M$ & $<500$ & VZV & Bilateral & 0 & 36 \\
\hline 16 & 32 & Asiatic & M & 160 & VZV & Bilateral & 0 & 120 \\
\hline 17 & 55 & White & $M$ & 38 & VZV & Unilateral & 7 & 210 \\
\hline 18 & 27 & White & $\mathrm{F}$ & 4 & HSV & Bilateral & 0 & 180 \\
\hline 19 & 46 & White & M & 1 & VZV & Bilateral & 0 & 120 \\
\hline 20 & 31 & White & M & & VZV & Bilateral & 0 & 83 \\
\hline
\end{tabular}

8 hours), intravenous ganciclovir $(5 \mathrm{mg} / \mathrm{kg}$ twice daily for 2 weeks then $5 \mathrm{mg} / \mathrm{kg} /$ day) or foscarnet $180 \mathrm{mg} / \mathrm{kg} /$ day in two or three divided doses for 2 weeks, then $90-120 \mathrm{mg} / \mathrm{kg} /$ day), intravenous ganciclovir ( $5 \mathrm{mg} / \mathrm{kg} /$ day) plus foscarnet $(90-120 \mathrm{mg} / \mathrm{kg} /$ day). If a patient was treated with ganciclovir and foscarnet individually, but not in combination, the drug used for the longest period of time was defined as maximum therapy. Patients were not necessarily treated with each regimen in a stepwise fashion. In most cases maximum therapy was begun at the time of diagnosis, and in the remainder therapy was rapidly escalated to maximum therapy over a period of 2 weeks or less following diagnosis. As clinical experience on this cohort accumulated, patients who presented later in the study received more aggressive treatment. This typically consisted of intravenous ganciclovir and foscarnet therapy.

The final visual acuity of all patients was the vision recorded at the last follow up visit. Decimal equivalents of Snellen visual acuities and standard values for counting fingers (0.01), hand movements (0.005), and light perception $(0.001)$ were utilised for statistical analysis. Outcomes were analysed for affected eyes $(n=39)$, and for patients $(n=20)$ using the mean acuity of the right and left eye. KruskallWallis non-parametric one way analysis of variance (KWAOV) was utilised to evaluate the efficacy of various forms of medical therapy in relation to the final visual outcome of these patients and eyes. $\chi^{2}$ analysis was used for comparisons of categorical data. Demographic variables and characteristics of clinical presentation and their effects on final visual outcome were also assessed using similar methods.

\section{Results}

Demographic data for the 20 patients is summarised in Table 1 . The mean patient age was 37.5 years. There were 17 males and three females. Of the 20 patients, 10 were white, six African-American, three Hispanic, and one Asian. The median CD4 count of the 18 patients for whom it was available was 21 cells $\times 10^{6} / 1$ with a range of 1 to 168 cells $\times 10^{6} / 1$.
The median duration of follow up of these patients was 180 days ( 6 months) with a range of 1.3 to 26 months. None of these demographic characteristics showed a statistically significant association with initial or final visual acuity.

Ophthalmic findings at baseline and treatment regimen for each patient are summarised in Table 2. The visual outcomes stratified by medical therapy are presented in Figure 1. The majority ( 15 of $20,75 \%$ ) of patients had a history of recent or ongoing VZV infections. Eleven patients had cutaneous zoster infection, one had herpes zoster ophthalmicus, two had central nervous system zoster meningitis or vasculitis, and one had disseminated zoster viraemia. Three patients had previous or concurrent herpes simplex virus (HSV) infections, two with HSV labialis and one with disseminated HSV infection. One patient (patient 14, Table 2) had cultures of her encrusted lip lesion which revealed HSV-1, and cultures and electron microscopic examination of a chorioretinal biopsy specimen from the more severely involved eye revealed herpes group viral particles which could not be subclassified. Patients 8 and 9 (Table 2) also underwent diagnostic vitrectomy and chorioretinal biopsy in one eye. The chorioretinal biopsy of patient 8 revealed no organisms on light microscopy and no growth of organisms on bacterial, viral, or fungal cultures. A diagnostic enucleation was subsequently performed which revealed complete retinal necrosis with no evidence of viral particles on light and electron microscopy. Diagnostic chorioretinal biopsy of the right eye of patient 9 revealed retinal necrosis and presence of VZV particles in the retina detected by immunoperoxidase staining. Some patients presented with more than one manifestation of VZV or HSV infection. Only two patients had no manifestations of extraocular VZV or HSV infections. Eleven of the 20 patients were being treated with acyclovir at the time of presentation for past or ongoing HSV or VZV infection. One patient was receiving intravenous maintenance foscarnet for inactive cytomegalovirus (CMV) retinitis. Eight patients $(40 \%)$ were not 
Table 2 Summary of treatment and visual outcome of 20 patients with varicella zoster virus (VZV) retinitis

\begin{tabular}{|c|c|c|c|c|c|c|c|}
\hline Patient & Eye & Initial vision & Therapy & $\begin{array}{l}\text { Retinal } \\
\text { detachment }\end{array}$ & $\begin{array}{l}\text { Time to detachment } \\
\text { (days) }\end{array}$ & Surgery & $\begin{array}{l}\text { Final } \\
\text { vision }\end{array}$ \\
\hline \multirow[t]{2}{*}{1} & $\mathrm{R}$ & $20 / 30$ & GCV & No & & Laser barrier & $20 / 40$ \\
\hline & $\mathrm{L}$ & $20 / 100$ & & Yes & 17 & PPV + silicone oil & $\mathrm{CF}$ \\
\hline 2 & $\mathrm{R}$ & $20 / 40$ & FOSC & Yes & 0 & $\mathrm{PPV}+$ gas & $\mathrm{CF}$ \\
\hline \multirow[t]{2}{*}{3} & $\mathrm{R}$ & $20 / 30$ & FOSC & Yes & 9 & PPV + silicone oil & NLP \\
\hline & $\mathrm{L}$ & $20 / 40$ & & Yes & 37 & PPV + silicone oil & $\mathrm{HM}$ \\
\hline \multirow[t]{2}{*}{4} & $\mathrm{R}$ & $20 / 20$ & $\mathrm{FOSC}+\mathrm{GCV}$ & Yes & 17 & PPV + silicone oil & $20 / 200$ \\
\hline & $\mathrm{L}$ & $20 / 20$ & & Yes & 40 & PPV + silicone oil & $20 / 60$ \\
\hline \multirow[t]{2}{*}{5} & $\mathrm{R}$ & $20 / 25$ & $\mathrm{FOSC}+\mathrm{GCV}$ & Yes & 17 & Laser barrier & $\mathrm{CF}$ \\
\hline & $\mathrm{L}$ & $20 / 25$ & & Yes & 26 & Laser barrier & $\mathrm{CF}$ \\
\hline \multirow[t]{2}{*}{6} & $\mathrm{R}$ & $20 / 200$ & $\mathrm{FOSC}+\mathrm{GCV}$ & Yes & 27 & PPV + silicone oil & HM \\
\hline & $\mathrm{L}$ & $20 / 400$ & & Yes & 132 & Laser barrier & LP \\
\hline \multirow[t]{2}{*}{7} & $\mathrm{R}$ & $20 / 20$ & $\mathrm{FOSC}+\mathrm{GCV}$ & Yes & 53 & PPV + silicone oil & $20 / 400$ \\
\hline & $\mathrm{L}$ & $20 / 40$ & & No & & None & HM \\
\hline \multirow[t]{2}{*}{8} & $\overline{\mathrm{R}}$ & $20 / 20$ & FOSC & No & & PPV - for biopsy & NLP \\
\hline & $\mathrm{L}$ & $20 / 25$ & & No & & None & NLP \\
\hline \multirow[t]{2}{*}{9} & $\mathrm{R}$ & $20 / 30$ & $\mathrm{FOSC}+\mathrm{GCV}$ & No & & PPV - for biopsy & NLP \\
\hline & $\mathrm{L}$ & $20 / 25$ & & Yes & 57 & PPV + silicone oil & $20 / 400$ \\
\hline \multirow[t]{2}{*}{10} & $\mathrm{R}$ & $20 / 200$ & $\mathrm{FOSC}+\mathrm{GCV}$ & Yes & 35 & PPV + silicone oil & NLP \\
\hline & $\mathrm{L}$ & $\mathrm{HM}$ & & No & & None & NLP \\
\hline \multirow[t]{2}{*}{11} & $\mathrm{R}$ & $20 / 400$ & $\mathrm{FOSC}+\mathrm{GCV}$ & Yes & 26 & PPV + silicone oil & $\mathrm{CF}$ \\
\hline & $\mathrm{L}$ & NLP & & No & & None & NLP \\
\hline \multirow[t]{2}{*}{12} & $\mathrm{R}$ & $20 / 40$ & ACV & Yes & & None & NLP \\
\hline & $\mathrm{L}$ & $20 / 40$ & & No & & None & NLP \\
\hline \multirow[t]{2}{*}{13} & $\mathrm{R}$ & $20 / 200$ & GCV & Yes & 14 & Laser barrier & $\mathrm{CF}$ \\
\hline & $\mathrm{L}$ & $20 / 400$ & & Yes & 14 & None & NLP \\
\hline \multirow[t]{2}{*}{14} & $\mathrm{R}$ & $\mathrm{LP}$ & $\mathrm{FOSC}+\mathrm{GCV}$ & No & & PPV - for biopsy & NLP \\
\hline & $\mathrm{L}$ & $20 / 30$ & & Yes & NA & None & $20 / 40$ \\
\hline \multirow[t]{2}{*}{15} & $\mathrm{R}$ & LP & ACV & No & & None & LP \\
\hline & $\mathrm{L}$ & $\mathrm{HM}$ & & Yes & 21 & None & NLP \\
\hline \multirow[t]{2}{*}{16} & $\mathrm{R}$ & $20 / 20$ & $\mathrm{FOSC}+\mathrm{GCV}$ & Yes & 73 & PPV + silicone oil & $20 / 400$ \\
\hline & $\mathrm{L}$ & $20 / 20$ & & Yes & 28 & Laser barrier & $20 / 30$ \\
\hline \multirow[t]{2}{*}{17} & $\mathrm{R}$ & $\mathrm{CF}$ & GCV & No & & None & NLP \\
\hline & $\mathrm{L}$ & $20 / 20$ & & Yes & 82 & PPV + silicone oil & $20 / 400$ \\
\hline \multirow[t]{2}{*}{18} & $\mathrm{R}$ & $20 / 20$ & ACV & Yes & 42 & PPV + silicone oil & NLP \\
\hline & $\mathrm{L}$ & $20 / 20$ & & Yes & 42 & PPV + silicone oil & NLP \\
\hline \multirow[t]{2}{*}{19} & $\mathrm{R}$ & $20 / 30$ & $\mathrm{ACV}$ & Yes & 12 & PPV + silicone oil & NLP \\
\hline & $\mathrm{L}$ & $20 / 60$ & & Yes & 12 & None & NLP \\
\hline \multirow[t]{2}{*}{20} & $\mathrm{R}$ & $20 / 20$ & $\mathrm{ACV}$ & Yes & 30 & None & NLP \\
\hline & $\mathrm{L}$ & $20 / 400$ & & Yes & 30 & None & NLP \\
\hline
\end{tabular}

$\mathrm{CF}=$ counting fingers; $\mathrm{HM}=$ hand movements; NLP = no light perception; $\mathrm{LP}=$ light perception; $\mathrm{GCV}=$ ganciclovir $; \mathrm{FOSC}=$ foscarnet; PPV = pars plana vitrectomy.

receiving any antiviral therapy at the time of presentation.

Ocular findings at the time of presentation included bilateral involvement in 14 of the 20 patients $(70 \%)$. Five of six of the unilateral cases $(83 \%)$ became bilateral less than 30 days after presentation. One patient remained unilateral after 6 months of follow up. Nearly all patients (17 of $20,85 \%)$ presented with peripheral lesions. Three patients $(15 \%)$ presented with both macular and peripheral lesions. These macular lesions demonstrated characteristic whitened, necrotic parafoveal outer retina, giving the appearance of a cherry red spot. Two of these patients had bilateral foveal involvement on presentation. Two other patients presented with peripheral lesions but within 5 months of presentation developed characteristic bilateral macular lesions which did not appear to be due to contiguous spread of peripheral retinitis. Neither macular nor peripheral presentation appeared to correlate with final visual acuity (KWAOV, $\mathrm{p}=0.37$ ). Control of retinitis was characterised by the lack of posterior progression and clearing of retinal opacification. Progression of retinitis was marked by new macular lesions or $200 \mu \mathrm{m}$ or more of posterior progression of punctate white outer retinal lesions or confluent white patches of outer retinal necrosis. Both retinal drawings and photographs were utilised in determining progression. Progression was often rapid and occurred in days compared with the slower progression of cytomegalovirus retinitis. Recurrence of retinitis was marked by new macular lesions or new outer retinal, white, punctate lesions posterior to the border of previously inactive retinitis.

The medical regimen initiated was intravenous acyclovir in nine patients, intravenous foscarnet in seven, intravenous ganciclovir in one, and combination daily foscarnet and ganciclovir in three of the 20 patients. The maximum medical treatment with which patients were treated included foscarnet and ganciclovir in nine patients, foscarnet in three patients, ganciclovir in three patients, and acyclovir in five patients. Toxicity from combination and monotherapy for ganciclovir, foscarnet, and acyclovir was not reported in any of the patients in this series. The side effects of monotherapy and combination therapy used in the treatment of CMV retinitis in patients with AIDS are well known and have been published elsewhere. ${ }^{5}$

Analysis of the initial visual acuities of the various medical treatment groups did not reveal any statistically significant differences among the four groups (KWAOV, $\mathrm{p}=0.74$ ). Analysis of final visual acuity based on the four methods of maximum medical treatment revealed that a statistically significant difference existed among the four treatment groups. The statistical conclusions were similar when analysed for each eye $(n=39, \mathrm{KWAOV}, \mathrm{p}=$ $0.0032)$ and for the average of the right and left eye visual acuity for each patient $(n=20$, KWAOV, $\mathrm{p}=0.0051)$. As an additional confir- 


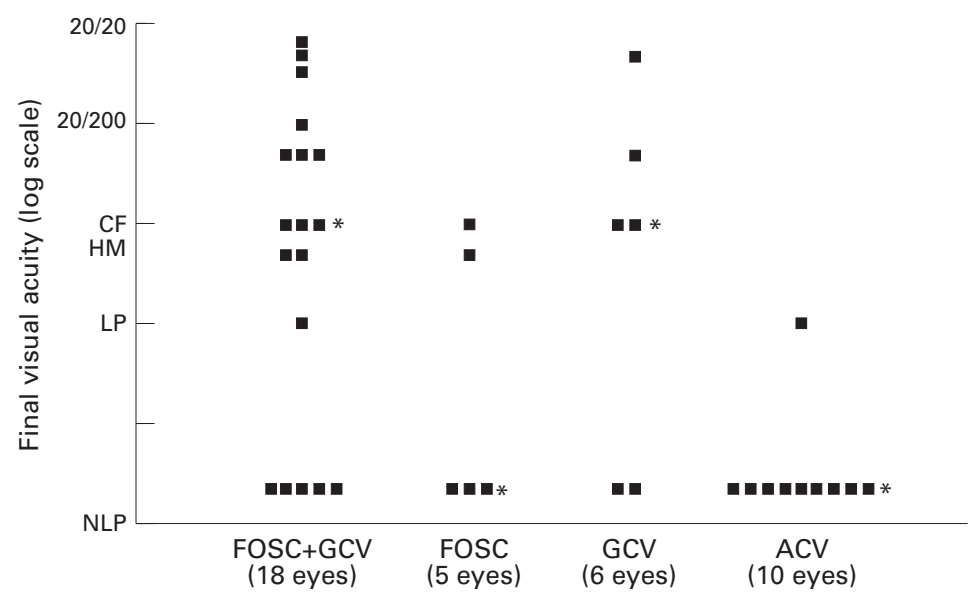

Figure 1 Visual outcomes of 39 eyes with varicella zoster virus retinitis segregated by maximum antiviral therapy. (FOSC $=$ foscarnet, $G C V=$ ganciclovir, $A C V=$ acyclovir; $\star=$ median values).

\section{Discussion}

In $1987 \mathrm{Jabs}$ and colleagues ${ }^{6}$ described three immunosuppressed patients with presumed varicella zoster retinitis. One of these patients had AIDS, cutaneous zoster, and retinitis that was poorly responsive to available antiviral therapy. Forster and colleagues ${ }^{1}$ later extended the earlier observations of Jabs and colleagues, ${ }^{6}$ and coined the term PORN in two patients with AIDS. Since then, one large series, several small series, and several case reports describing this disease have appeared in the literature. Only anecdotal information is available to assist in the medical management of this devastating disease.

PORN is frequently compared and contrasted with acute retinal necrosis (ARN). ARN is an acute necrotising viral retinitis associated with moderate to severe vitritis and retinal vasculitis that classically occurs in otherwise healthy patients. ${ }^{7}$ The diagnostic criteria of ARN, as enumerated by the American Uveitis Society, have been expanded to include immunocompromised patients, but exclude patients without a '...prominent inflammatory reaction in the vitreous and anterior chamber'. ${ }^{8}$ Bilaterality with ARN is relatively uncommon at the time of presentation. ARN is usually caused by activation of latent VZV infection, but cases caused by HSV have been well documented. Treatment with acyclovir, the drug of choice in ARN, is often effective in controlling the retinitis and reducing the risk of involvement of the other eye. PORN is a syndrome that affects immunocompromised patients, particularly those with AIDS..$^{1-46}{ }^{10}{ }^{11}$ Friedman and colleagues ${ }^{12}$ noted that VZVR may also be the first manifestation of AIDS. All of the patients in this series met the diagnostic criteria for PORN, ${ }^{3}$ but not for $\mathrm{ARN}{ }^{\text {? }}$

Varicella zoster virus has been implicated as the cause of PORN. Forster and colleagues ${ }^{1}$ demonstrated electron microscopic evidence of viral particles consistent with a herpes group virus in one patient, and polymerase chain reaction (PCR) techniques suggested the presence of a similar virus in another. Margolis and colleagues $^{2}$ demonstrated VZV in vitreous or retinal cultures, by immunohistochemical techniques, and by electron microscopy in three of five patients with PORN. Kupperman and colleagues ${ }^{4}$ also demonstrated VZV DNA using PCR techniques on a retinal biopsy specimen from one AIDS patient with VZVR. Using immunohistochemical methods, they also demonstrated zoster antigens in the retina, but not the choroid, in an enucleated eye from another patient with VZVR. ${ }^{4}$ Furthermore, most patients with PORN demonstrate evidence of past or ongoing cutaneous or disseminated VZV infection. ${ }^{1-4}$ This is uncommon in immunocompetent individuals with ARN. Kupperman and colleagues ${ }^{4}$ also demonstrated that two of their six patients with VZVR had evidence of VZV mediated central nervous system vasculitis or meningitis. In our series, two patients had VZV mediated central nervous system vasculitis or meningitis. This body of direct and indirect evidence convincingly points to VZV as a cause of PORN syndrome cataract extraction and posterior chamber intraocular lens implantation 17 months after presentation. 
among patients with AIDS. In our series, however, three patients presented with apparent previous or concurrent HSV infections with no history or evidence of VZV infections. Despite atypical extraocular characteristics, these patients had an appearance and clinical course indistinguishable from other patients with PORN. Cutaneous HSV infections are, however, quite common in patients with AIDS. Their concurrent presence does not imply that HSV is the causative agent for PORN. Although none of the biopsy specimens from our series demonstrated HSV particles in the retina, it is still possible that, like ARN, VZV or, far more rarely, HSV may cause VZVR.

The distinguishing features of PORN include a propensity for bilateral involvement, absent or minimal inflammatory response, very rapid progression, and a poor response to acyclovir. ${ }^{1-4}$ Patients usually present with punctate peripheral lesions alone that coalesce anteriorly with or without posterior pole involvement. One report of five patients emphasised early posterior pole involvement as a feature which distinguished PORN from $\mathrm{ARN}^{2}$; however, in a recent report of 38 patients only one third presented with posterior pole disease. ${ }^{3}$ Involvement of the posterior pole typically occurs by spread of the peripheral lesions. There is a very characteristic macular lesion seen in some patients with VZVR. These patients have an abrupt loss of central vision and demonstrate opacification of the paracentral outer retina, with a foveal 'cherry red spot'. These macular lesions are not contiguous with the peripheral retinitis. ${ }^{12}$ We have seen these lesions occur as the presenting symptom or, later, after the initiation of medical therapy.

There tends to be rapid progression of the disease over the course of days to weeks. The peripheral lesions rapidly progress posteriorly. As the peripheral retinitis burns out, retinal atrophy and retinal pigment epithelial disturbances appear. Initial perivascular involvement followed by perivascular retinal atrophy with adjacent areas of active retinitis give the appearance of perivascular clearing.

Forster $^{1}$ and later Johnston and associates ${ }^{11}$ showed a poor response to intravenous high dose acyclovir. Engstrom and associates ${ }^{3}$ described 38 AIDS patients with the diagnosis of PORN. Acyclovir was used as the sole therapy in the majority of the patients. Visual acuity was NLP in two thirds of the eyes within 4 weeks of diagnosis. Our results are slightly better with final visual acuity of NLP in $49 \%$ of eyes after a median follow up period of 6 months. The better visual outcome in the current series may be due to the more frequent use of antivirals other than acyclovir. Acyclovir was used as the primary mode of therapy in 26 of 38 patients $(68 \%)$ in Engstrom's series ${ }^{3}$ compared with five of $20(20 \%)$ patients in the present series. In our series nine of 10 eyes $(90 \%)$ treated with acyclovir alone had final vision of NLP, whereas 10 of 29 eyes (34\%) treated with other antivirals had final vision of $\mathrm{NLP}\left(\chi^{2}, p=0.0078\right)$. Most of these were treated with combination therapy. The rationale for combination antiviral therapy in VZVR was derived in part from the results of Studies of Ocular Complications of AIDS Research Group $^{5}$ in the treatment of CMV retinitis with both ganciclovir and foscarnet, and from other authors who suggested combination antiviral therapy for the treatment of VZVR. ${ }^{1311}$ Analysis of our data suggests that therapy with combination foscarnet and ganciclovir may reduce visual loss in patients with VZVR. The small numbers of patients in the ganciclovir group and the foscarnet group make us reluctant to generalise any analysis of these groups.

Because of previous cutaneous or disseminated zoster infections, many AIDS patients are on maintenance acyclovir therapy at the time of presentation with VZVR. In our series 11 of 20 patients were receiving oral acyclovir therapy at the time of presentation. Although oral acyclovir maintenance doses against nonocular VZV may be lower than induction doses for VZV retinitis, it is possible that the development of resistance of the VZV to acyclovir may contribute to the poor response to therapy with intravenous acyclovir. Jacobson and colleagues ${ }^{13}$ suggested that acyclovir resistance and an associated massive viraemia may arise from an inadequate dosing regimen of acyclovir owing to poor patient compliance or poor bioavailability of the drug. Pavesio and colleagues $^{14}$ have suggested that intravitreal antivirals may also be useful in the management of VZVR since high levels of ganciclovir and foscarnet can be attained in the vitreous cavity and retina. Although this was not the primary focus of our study, combined intravenous and intravitreal antiviral therapy may be an efficacious method of controlling viral replication in the early active phases of the disease. Further studies are required to determine the exact role of intravitreal antivirals in the treatment of VZVR.

The two main vision threatening complications of VZVR are extensive retinal necrosis and RRDs. ${ }^{1-3}$ Engstrom and colleagues ${ }^{3}$ have reported that $70 \%$ of 51 eyes with PORN developed RRDs, usually within 2 months of presentation. In our series, RRDs developed in 27 of 39 eyes $(69 \%)$ within a median period of 30 days of presentation. Among the 20 eyes which had surgery for RRD, 14 underwent pars plana vitrectomy with silicone oil tamponade, two eyes underwent pars plana vitrectomy and intraocular gas tamponade, and five eyes underwent laser barrier photocoagulation. On final follow up, $87 \%$ of eyes that underwent pars plana vitrectomy and silicone oil tamponade for RRD remained attached. Our data indicate that surgery is capable of anatomic success and stabilisation of vision.

PORN is a viral retinitis syndrome usually caused by VZV, and perhaps HSV, that occurs in patients with AIDS. Evidence of recent or concurrent herpes zoster infections in immunocompromised patients with retinitis should suggest a diagnosis of PORN. Cytomegalovirus retinitis remains the most common opportunistic retinal infection in people with AIDS, and VZV a distant second. These two infections are distinct in their appearance and clinical course 
and should rarely present a diagnostic dilemma for experienced examiners.

PORN has a dismal prognosis with a propensity for bilateral involvement and severe loss of vision. Our study is unique in its relatively long follow up and in its analysis of the visual outcomes of various modes of medical therapy. This study is limited by its non-randomised, non-blinded design. However, the analysis for confounding variables, and the high statistical confidence of the analysis of visual outcomes strongly suggest that aggressive antiviral therapy improves the visual prognosis in patients with VZVR. Acyclovir appears to be relatively ineffective. We currently recommend concurrent therapy with ganciclovir and foscarnet. We hope these results will further stimulate clinical trials to improve the understanding and treatment of this disease.

This work was supported in part by an unrestricted grant from Research to Prevent Blindness Inc, New York, USA.

1 Forster DJ, Dugel PU, Frangieh GT, Liggett PE, Rao NA. Rapidly progressive outer retinal necrosis in the acquired

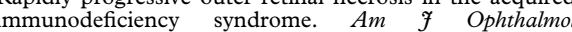
1990;110:341-8.

2 Margolis TP, Lowder CY, Holland GN, Spaide RF, Logan AG, Weissman SS, et al. Varicella-zoster virus retinitis in patients with the acquired immunodeficiency syndrome. Am F Ophthalmol 1991;112:119-31.

3 Engstrom RE Jr, Holland GN, Margolis TP, Muccioli C, Lindley JI, Belfort R Jr, et al. The progressive outer retinal retinopathy in patients with AIDS. Ophthalmology 1994; 101:1488-502.
4 Kupperman BD, Quiceno JI, Wiley C, Hesselink J, Hamilton R, Keefe K, et al. Clinical and histopathologic study of varicella zoster virus retinitis in patients with the acquired immunodeficiency syndrome. Am $\mathcal{f}$ Ophthalmol 1994;118:589-600.

5 The Studies of Ocular Complications of AIDS Research Group in collaboration with the AIDS Clinical Trials Group. Combination foscarnet and ganciclovir therapy vs monotherapy for the treatment of relapsed cytomegalovirus retinitis in patients with AIDS. The cytomegalovirus retreatment trial. Arch Ophthalmol 1996;114:23-33.

6 Jabs DA, Schachat AP, Liss R, Knox DL, Michels RG. Presumed varicella zoster retinitis in immunocompromised patients. Retina 1987;7:9-13.

7 Urayama A, Yamada N, Sasaki T, Nishiyama Y, Watanabe $\mathrm{H}$, Wakusawa $\mathrm{S}$, et al. Unilateral acute uveitis with retinal periarteritis and detachment. $\mathcal{f} p n$ f Clin Ophthalmol 1971;25:607-19.

8 Holland GN, and the Executive Committee of American Uveitis Society. Standard diagnostic criteria for the acute retinal necrosis syndrome. Am f Ophthalmol 1994;117:6637.

9 Chess J, Marcus DM. Zoster-related bilateral acute retinal necrosis syndrome as presenting sign of AIDS. Ann Ophthalmol 1988;20:431-5.

10 Duker JS, Shakin EP. Rapidly progressive outer retinal necrosis in the acquired immunodeficiency syndrome. [Letter; comment] Am f Ophthalmol 1991;111:255-6.

11 Johnston WH, Holland GN, Engstrom RE Jr, Rimmer S. Recurrence of presumed varicella-zoster virus retinopathy in patients with acquired immunodeficiency syndrome. $\mathrm{Am}$ in Ophthalmol 1993;116:42-50.

12 Friedman SM, Margo CE, Connelly BL. Varicella-zoster virus retinitis as the initial manifestation of the acquired immunodeficiency syndrome. Am f Ophthalmol 1994;117: 536-8.

13 Jacobson MA, Berger TG, Fikrig S, Becherer P, Moohr JW, Stanat SC, et al. Acyclovir-resistant varicella zoster virus infection after chronic oral acyclovir therapy in patients with acquired immunodeficiency syndrome (AIDS). Ann Intern Med 1990;112:187-91.

14 Pavesio CE, Mitchell SM, Barton K, Schwartz SD, Towler HM, Lightman S. Progressive outer retinal necrosis (PORN) in AIDS patients: a different appearance of varicella-zoster retinitis. Eye 1995;9:271-6. 